\title{
Session 9: Manufacturing
}

\author{
Friday 16th April 2010. Moderator: Gregory T. Bleck
}

[08.30-09.15]

'The business of antibodies - how to protect and commercialize antibodies'

Andrew T. Serafini

Fenwick and West LLP, USA

Abstract not available.

[09.15-09.45]

'cDNA to antibody candidate selection to a 3$5 \mathrm{~g} / \mathrm{L}$ therapeutic antibody production process in six months'

Gregory T. Bleck

Catalent Pharma Solutions, Middleton, Wisconsin, USA

A consistent procedure to allow antibody candidate screening on the path to a high expressing therapeutic antibody cell line is critical for quickly moving antibodies from discovery to clinical trials. The GPEx ${ }^{\circledR}$ method allows different antibody variants or subclasses (mg to gram quantities) to be produced for characterization and screening 10-12 weeks after start of development from a pool of clonal cell lines. Selection of the therapeutic candidate occurs after analysis of the various $\mathrm{CHO}$ produced antibodies, and clonal cell lines are developed for the lead molecule. The resulting clonal cell lines have specific productivities ranging between 30 and 100 picograms/cell/day and final antibody titers of 3-5 g/L in the selected culture process. Our experience making $\mathrm{CHO}$ cell lines producing over 150 different antibodies will be summarized and case studies of antibody cell line development will be discussed.

[09.45-10.05]

'Manufacturing human antibodies for preclinical research and market, today and tomorrow'

Florian M. Wurm and Maria De Jesus

Swiss Federal Institute of Technology, Lausanne, Switzerland
Specific antibodies or antibody-fusion proteins are produced now in $\mathrm{kg}$ to ton quantities for the treatment of a small number of diseases, benefiting tens of thousands of affected patients. The unprecedented medical and commercial success of these molecules has raised enormous interest in the biopharmaceutical research community, resulting in hundreds of new molecules to be explored for the same or other diseases. A major bottleneck for bringing such promising protein candidates for treatment into the clinical setting is the amount of time and money required to manufacture even gram quantities of these under appropriate conditions with reproducible yield and high quality. Mammalian cells in bioreactors have been used and optimized over the last 25 years to produce these antibodies with ever increasing yields. Today, gram/liter quantities of high quality antibody preparations can be made in reactors of up to 20'000 Liter volume. However, each molecule is a new challenge and requires profound development efforts to obtain such highly productive production processes. The knowledge for such process development and establishment of manufacturing capacity is in the hands of few leading companies. The talk will cover the evolution of insights and processes during the last 2 decades and will also provide a view on the future in process sciences and manufacturing of antibodies and other recombinant antibodies made in mammalian cells in bioreactors. An emphasis will be given on fast, efficient and new production principles, such as large scale transient gene expression and the use of single use, disposable bioreactor systems. All of these are able to cut cost and to reduce time for moving a new expression vector to the reality of sufficient quantity of product for preclinical and clinical research, as well as for manufacturing for the market. 
[10.05-10.25]

'Characterization of the Wave bioreactor: Residence time distribution determination'

M. Elisa Rodrigues, A. Rita Costa, Mariana Henriques, Joana Azeredo and Rosário Oliveira

Universidade do Minho, Braga, Portugal

The increasing importance of therapeutic antibodies to modern medicine and their high clinical dose requirements have led to the development of technologies that aim to increase biomanufacturing capacity. Most of these technologies focus on mammalian cell culture, and several bioreactors are currently available either for adherent or suspension culture. Among them, disposable reactors have been attracting increased attention in the last years, particularly the Wave bioreactor. In this system, an undulation movement is induced to the cell culture, ensuring good mixing and oxygen transfer without causing shear damage to the cells. Furthermore, this reactor requires no cleaning or sterilization, providing ease of operation and protection against cross-contamination. However, since Wave is still a recent technology, further characterization needs to be performed so that the know-how becomes comparable to that of more common bioreactors (i.e. stirred tank reactor (STR)). In this sense, an evaluation of the residence time distribution (RTD) in a Wave reactor was performed, in order to characterize its mixing and flow and to compare its behavior with ideal models and a commercial STR available for mammalian cell culture. For this, RTD was determined using methylene blue with a pulse input methodology, at three flow rates, usually identified as low (L: $3.3 \times 10^{-5} \mathrm{~m}^{3} / \mathrm{h}$ ), intermediate (I: $\left.7.9 \times 10^{-5} \mathrm{~m}^{3} / \mathrm{h}\right)$, and high $\left(\mathrm{H}: 1.25 \times 10^{-4}\right.$ $\mathrm{m}^{3} / \mathrm{h}$ ) for mammalian cell culture. Samples were taken periodically and the absorbance read at $660 \mathrm{~nm}$. The results obtained show that the behavior of Wave approximates the ideal and experimental continuous STR at flow rate L. For flows I and $\mathrm{H}$, the least square fitting of data from Wave indicates a considerable deviation from the ideal models.

Additionally, the comparison of the average residence time $(\mathrm{tr})$ with the time of passage $(\tau)$ of both Wave and STR provided a possible explanation for the non-ideality of their behavior. For STR, tr was lower than $\tau$ for all the flow rates tested, indicating the development of dead zones inside the reactor. The same was observed at flow rate $\mathrm{H}$ in Wave. However, for flows $\mathrm{L}$ and $\mathrm{I}$, the deviation from the ideal behavior can be a result of the development of short-circuits inside the reactor, as indicated by a tr higher than $\tau$.

In conclusion, this study demonstrated that the choice of the flow rate will strongly influence the behavior of the Wave bioreactor. The use of a low flow rate seems to be a choice that provides a behavior closer to an ideal model (continuous STR). 